\title{
Analysis of intra-pulse frequency-modulated, low probability of interception, radar signals
}

\author{
A R SACHIN*D, SOORAJ K AMBAT and K V S HARI \\ Department of Electrical Communication Engineering, Indian Institute of Science, Bangalore 560012, India \\ e-mail: sachin@dare.drdo.in; sooraj@npol.drdo.in; hari@ece.iisc.ernet.in
}

MS received 22 January 2016; revised 16 January 2017; accepted 16 January 2017

\begin{abstract}
In this paper, we investigate the problem of analysis of low probability of interception (LPI) radar signals with intra-pulse frequency modulation (FM) under low signal-to-noise ratio conditions from the perspective of an airborne electronic warfare (EW) digital receiver. EW receivers are designed to intercept and analyse threat radar signals of different classes, received over large dynamic range and operating independently over large geographical spread to advice host aircraft to undertake specified actions. For an EW receiver, primary challenges in interception and analysis of LPI radar signals are low received power, intra-pulse modulations, multi-octave frequency range, wide signal bandwidth, long pulse width, vast and multi-parametric search space, etc. In the present work, a method based on match filterbank localization and Taylor's series approximation for analysing the entire family of intra-pulse FM radar signals is proposed. The method involves progressive, joint time-frequency (TF) localization of the signal of interest (SOI), under piecewise linearity and continuity assumptions on instantaneous frequency, to effectively capture local TF signatures. Detection is by information-theoretic criterion based hypotheses testing, while estimation and classification are based on polynomial approximation. Fine signal analysis is followed by synthetic reconstruction of the received signal slope. Detection, estimation and classification performances for the prominent FM radar signal classes are quantified based on simulation study statistics. Stagewise implementation of analysis and FM slope reconstruction, in realistic radar threat scenarios, is demonstrated for the potential SOIs. Subject discussion is organized from the perspective of practical EW system design and presented within the realm of signal processing architecture of concurrent EW digital receivers.
\end{abstract}

Keywords. Digital receiver (DRx); electronic warfare (EW); information theoretic criterion (IC); low probability of interception (LPI); minimum description length (MDL); time-bandwidth product (TBP).

\section{Introduction}

Modern electronic warfare (EW) receiver employs a host of concepts, algorithms, technologies, sensors and systems. It incorporates wide-open receivers for multi-octave spectral coverage and super-heterodyne digital receivers (DRx) for precision analysis [1]. Low probability of interception (LPI) radars are specially designed to defeat the basic EW function, i.e., signal interception, and adversely impact all stages of EW functionality. Wide-open receivers are ineffective against LPI radars due to high thermal noise. Highsensitivity DRx with wide signal bandwidth are used for LPI radar signal interception and analysis. In a typical scenario, received signal is a linear combination of multiple radar signals of various types like pulsed, pulse compressed, continuous wave (CW), LPI, etc., in additive, white, Gaussian noise (AWGN). To be relevant in today's electronic battlefield, EW systems should be capable of

*For correspondence handling dense threat scenarios of low signal to noise ratio (SNR), non-stationary, large time-bandwidth product (TBP) signals [2].

Commercially, prominent radar and EW system manufacturers like [3-6] specify LPI radar detection and intrapulse analysis as key features in modern EW systems, indicating growing prominence of LPI radars for military applications.

Pioneering work has been done by P E Pace in [7] with extensive discussion on the technological and operational aspects of LPI radars, signal design and analysis. A method based on parallel filter arrays and high-order statistics, presented in [8] addresses analysis of prominent LPI radar signal classes. Time-Frequency (TF) analysis methods have attracted considerable focus and many research works have been reported using different variants of TF distributions. In [9], authors have proposed system implementation of Choi-Williams distribution (CWD) and quadrature mirror filter bank. Extensive research for LPI radar signal classification based on artificial neural networks has been 
reported in [10]. Methods like spectrogram, Wigner-Ville distribution (WVD) and CWD have been used for signal transformation and representation [11]. The Polynomial phase signal modelling method in [12] demonstrates analysis performance for LFMCW signals. A novel method based on periodic Wigner-Ville Hough transform (WVHT) for LFMCW analysis has been proposed, with detailed analytical discussion in [13]. Information theoretic criterion (IC)-based methods have been widely referred to for model order selection and is adopted in the present work also for signal detection [14, 15]. An interesting method using short-time harmonic model and Kolmogorov-Smirnov test is presented in [16]. The authors have proposed a comprehensive solution for detection, characterization and geolocalization of LFMCW LPI signals. Analytical aspects of range-azimuth FMCW radar sensor using joint angle and delay estimation discussed in [17] gives a fine insight into FMCW radar signals. Sparse representation of intra-pulse modulated signals is presented in [18], with demonstrated performance for linear and non-linear frequency modulation (FM) using atomic dictionary. In [19], implementation of Wigner-Hough transform based detectors for LFMCW signals is discussed, along with a comparison of various detector configurations. Design aspects of low probability of recognition radar signals and radar performance as a function of covertness has been holistically presented in [20]. In [21], wavelet-packet based method to analyse FM LPI radar signals is proposed, along with performance comparison with CWD and Fourier transform.

A detailed treatment of the subject, based on the principles of atomic decomposition, expectation maximization, iterative recovery and IC, has been reported in [22-24]. The Authors have presented a host of theoretical foundations, performance bounds and comparative studies addressing various aspects of analysis. The present work has some similarities with these papers. However, it is designed for a different set of problems for a broader class of signals of interest (SOIs). In [22], authors have proved analysis optimality for linear chirplets. However, SOIs with nonlinear or periodic slopes have infinite parametric search space. Localization for such signals within true parametric neighbourhood using linear chirplet models, in a multi parametric search space, is a practical concern. Assuming good localization, next difficulty is uncertainty in the received signal structure, since interception is non-cooperative and arbitrary structures are possible. Even for a single non-linear SOI, the solution obtained is not optimal and iterative signal extraction is likely to be affected by residues, more so in a large dynamic range system. The present work addresses the problems associated with analysis under non-linear, periodic, aperiodic and arbitrary instantaneous frequency (IF) constraints. The method in [22] uses chirplet dictionary with reported analysis of linear chirplets. The proposed solution uses a compact match filterbank (MFB) for localization of entire family of intrapulse FM signals. The analysis is designed for any pulse width (PW) above a minimum reconfigurable value, process during reception and analysis of SOIs, without constraints on observation vector length, periodicity or linearity of IF of SOIs.

Notations: Bold upper case and bold lower case Roman letters denote matrices and vectors, respectively. $\mathbf{x}$ is a column vector. $\hat{\mathbf{x}}, \mathbf{x}^{H}$ and $\tilde{\mathbf{x}}$ denote estimate, hermitian and error-vector of $\mathbf{x}$, respectively. $\|$.$\| denotes vector 2$-norm. $\perp$ denotes orthogonality. $\sim$ denotes 'distributed as'. $\mathcal{N}$ and $\mathcal{C N}$ denote normal and complex normal distributions, respectively. $H_{0}$ and $H_{1}$ denote null and true hypotheses, respectively.

\section{Frequency modulated radar signals}

FM is a commonly used radar pulse compression technique [25] due to advantages like good pulse compression ratio, fine target resolution, low peak power, longer PW design, etc. Large TBP signals can be realized with linear/nonlinear FM (LFM/NLFM) slopes. These attributes facilitate mission-centric signal design, and make FM a suitable choice for LPI applications. The proposed solution is designed for following radar signal types:

- Unmodulated (Unmod),

- LFM,

- NLFM,

- Linear/Non-Linear Continuous Wave FM (FMCW) and

- Arbitrary FM (ArFM).

Discrete-time expression of an unmodulated radar signal is

$$
s[n]=(\alpha) \exp \{j[2 \pi F n+\varphi]\}=(\alpha) \exp \{j[\omega n+\varphi]\}
$$

where $j=\sqrt{-1}, \mathbf{s}$ is signal vector, $\alpha$ is amplitude, $F$ is centre frequency, $\varphi$ is initial phase offset and $n$ is time vector.

FM radar signal, whose IF is expressed as $q$ th order polynomial is represented by

$$
\begin{aligned}
s[n] & =(\alpha) \exp \left\{j\left[2 \pi\left(\left(F \mp \frac{B}{2}\right) n \pm \frac{B}{(q+1) T} n^{(q+1)}\right)+\varphi\right]\right\} \\
& =(\alpha) \exp \left\{j\left[\left(\omega_{q+1} n+\omega_{1} n^{(q+1)}\right)+\varphi\right]\right\}
\end{aligned}
$$

where $B$ is modulation bandwidth, $T$ is modulation period, $q$ is polynomial order and $\omega_{i}$ are FM parametric coefficients.

FM signals with IF approximated by higher order Taylor's series are referred to herein as ArFM and are given by

$$
s[n]=(\alpha) \exp \left\{j\left[\left(\omega_{4} n+\omega_{3} n^{2}+\omega_{2} n^{3}+\omega_{1} n^{4}\right)+\varphi\right]\right\} .
$$

FMCW radar signals have periodic IF with unique positive and negative slopes. Each unidirectional segment of 
FMCW waveform is represented by generic FM signal expression and may be symmetric or asymmetric. Positive and negative slopes may belong to same or different types with equal or unequal time periods. In the present work, LFM and NLFM with single, unidirectional slope are considered as basic structures. Unmod is treated as LFM with zero slope. Periodic linear/non-linear slopes of FMCW are decomposed into constituent basic structures and analysed with a standardized processing architecture. This architecture is applicable to ArFM CW signals also, without any assumption on the IF structure.

\section{Objectives, scope, and assumptions}

In this section, objectives, scope and assumptions of the present work are discussed. This gives an overview of the complexity of the problem being addressed, design philosophy followed and qualitative content of the proposed architecture.

\subsection{Objectives}

To design a method for the analysis of intra-pulse FM, LPI, threat radar signals from the perspective of an airborne EW digital receiver, under low SNR constraint for

1. detection of the signal,

2. estimation of the signal and modulation parameters,

3. classification of the signal based on modulation and

4. reconstruction of IF vector of the signal.

\subsection{Scope}

The proposed method holds good for analysis of SOIs with continuous time, linear/non-linear IF, including periodic, aperiodic, CW and arbitrary slopes. Analysis is designed for multiple, interleaved and non time-overlapped SOIs.

\subsection{Assumptions}

1. IF structure of $(L \times 1)$ SOI is piecewise continuous and approximately linear over $m$ samples, where $m \ll L$.

2. IF structure of $(L \times 1)$ SOI is piecewise continuous over $M$ samples, where $m<M<L$.

\section{Problem statement}

For interception analysis, RF band of interest is downconverted and digitized under Nyquist band-pass sampling regime. The received real process $\mathbf{b}_{r}$ is a linear combination of SOI and real AWGN, with zero mean and unknown variance $\frac{\sigma^{2}}{2}$ :

$$
\mathbf{b}_{\mathbf{r} L \times 1}=\mathbf{s}_{r}+\mathbf{w}_{r}, \quad \mathbf{w}_{r} \sim \mathcal{N}\left(\mathbf{0}, \frac{\sigma^{2}}{2} \mathbf{I}_{L}\right) .
$$

$\mathrm{SNR}$ definition for the received process $\mathbf{b}_{r}$ is given by the relation

$$
\mathrm{SNR}=10 \log _{10}\left[\frac{\left\|\mathbf{s}_{\mathrm{r}}\right\|^{2}}{0.5 \sigma^{2}}\right] \quad \mathrm{dB} .
$$

Analytic representation, $\mathbf{b}$, of the real vector, $\mathbf{b}_{r}$, is obtained by Hilbert transform (HT) of $\mathbf{b}_{r}$ and expressed as

$$
\mathbf{b}_{L \times 1}=\mathbf{s}+\mathbf{w}, \quad \mathbf{w} \sim \mathcal{C N}\left(\mathbf{0}, \sigma^{2} \mathbf{I}_{\mathbf{L}}\right),
$$

where $\mathbf{s}=\mathbf{s}_{r}+\mathbf{j} \mathbf{s}_{i}$ and $\mathbf{w}=\mathbf{w}_{r}+\mathbf{j} \mathbf{w}_{i} ; \mathbf{w}_{r} \perp \mathbf{w}_{i}$ implies that $\mathbf{w}_{r}$ and $\mathbf{w}_{i}$ are uncorrelated and independent [26]. The corresponding noise covariance matrix $\mathbf{R}_{\mathbf{w}}$ is computed using

$$
\mathbf{R}_{\mathbf{w}}=\mathbb{E}\left[\mathbf{w} \mathbf{w}^{H}\right]=\sigma^{2} \mathbf{I}_{L} .
$$

Probability density function (PDF) of an $(L \times 1)$, zeromean, complex, Gaussian vector satisfying circularity condition is

$$
p_{\mathbf{w}}=\frac{1}{\pi^{L}} \frac{1}{\left|\mathbf{R}_{\mathbf{w}}\right|} \exp \left[-\mathbf{w}^{H} \mathbf{R}_{\mathbf{w}}^{-1} \mathbf{w}\right] .
$$

Under hypotheses $H_{0}$ and $H_{1}$, PDFs of received process are

$$
\begin{gathered}
p\left(\mathbf{b} ; \Theta^{(0)}\right)=\frac{1}{\left(\pi \sigma^{2}\right)^{L}} \exp \left[\frac{-\|\mathbf{b}\|^{2}}{\sigma^{2}}\right], \quad \Theta^{(0)}=\left\{\sigma^{2}\right\} \\
p\left(\mathbf{b} ; \Theta^{(1)}\right)=\frac{1}{\left(\pi \sigma^{2}\right)^{L}} \exp \left[\frac{-\|\mathbf{b}-\mathbf{s}\|^{2}}{\sigma^{2}}\right], \quad \Theta^{(1)}=\left\{\boldsymbol{\Omega}, \sigma^{2}\right\}
\end{gathered}
$$

where $\boldsymbol{\Theta}^{(0)}$ and $\boldsymbol{\Theta}^{(1)}$ are parametric vectors under hypotheses $H_{0}$ and $H_{1}$, respectively. $\boldsymbol{\Omega}=\left\{\omega_{i}\right\}$ is the parametric vector of SOI. Objective function formulation for hypotheses testing is based on PDF maximization, i.e.,

$$
\hat{\boldsymbol{\Theta}}^{(1)}=\underset{\boldsymbol{\Theta}^{(1)}}{\arg \max }\left[p\left(\mathbf{b} ; \Theta^{(1)}\right)\right] .
$$

Maximizing the PDF in Eq. (11) is equivalent to minimizing sum of squares of error (SSE) vector by estimating $\hat{\Omega}$ using

$$
\hat{\Omega}=\underset{\mathbf{\Omega}}{\arg \min }\|\mathbf{b}-\mathbf{s}\|^{2} .
$$

If $H_{1}$ is indeed true, $\hat{\Omega}$ and corresponding $\hat{\mathbf{s}}$ are the optimal parametric and signal vector estimates, while $\hat{\sigma}^{2}$ is estimated using the residue. If $H_{0}$ holds, $\hat{\sigma}^{2}$ is computed using $p\left(\mathbf{b} ; \Theta^{(0)}\right)$. 


\section{Analysis architecture}

\subsection{Research gap and system considerations}

The literature referred to in this paper includes reputed publications on the subject over the last decade. It is seen that majority of the solutions employ TF methods like spectrogram, WVD, pseudo-WVD and CWD. Many novel solutions to specific LPI classes like LFMCW have been proposed. Specialized methods like atomic decomposition, wavelet packets, neural networks, WVHT, IC, etc. have also been reported.

From the perspective of a practical EW system, methods for a few specific SOI classes and/or methods for only specific functions like signal detection, although of theoretical significance, do not form a complete solution for field deployment. System requirement is for a comprehensive solution for different classes of SOIs, including unknown/arbitrary types and implementable with the current technologies and systems. It is also important that methods chosen for different functions should have optimal compatibility among them for seamless integration. Among the referred publications, each one offers highly valuable methods within their scope and focus, along with stand-alone performance. The current work is driven by system considerations, to design an unified solution for the entire FM class, by optimal combination of methods, and demonstrates integrated performance in potential scenarios.

\subsection{Overview of the proposed solution}

The Proposed analysis primarily comprises two stages, as shown in figure 1 . In the first stage, signal processing and detection is performed hierarchically starting from HT of input up to intra-pulse deinterleaving. If $H_{1}$ holds, pulse forming, deinterleaving of pulses belonging to different SOIs and FMCW identification/deinterleaving are carried out. In case of no signal detection, analysis stops with computation of noise variance. In the second stage, estimation and classification functions are performed in a conditional, decision tree structure. This stage is executed only if $H_{1}$ holds true and works on various results obtained in the first stage. A novel concept in the proposed architecture is synthesis of match-filter localized, short-duration, complex signal frames using Taylor's series estimate of phase angle vectors and using the same for signal detection. This enables processing using a common projection scheme, independent of underlying IF structure. The first level solution is to seek local estimates up to a predefined parametric resolution under linearity and continuity assumptions on IF of SOI. Fine analysis is done using initial estimates under continuity assumption only. This standardizes search process to linear IF and progressively constructs non-linear IF under least-square error minimization formulation. Functional description of different stages of analysis and process flow under conditionalities is presented next.

\subsection{Pre-processing and $H T$}

The objective in these steps is robust TF localization of SOI. The proposed piecewise processing is similar in concept to short-time Fourier transform (STFT). STFT projects SOI onto harmonic sequence of each frequency, whereas the proposed method projects SOI onto match function vectors for spectral localization, for better

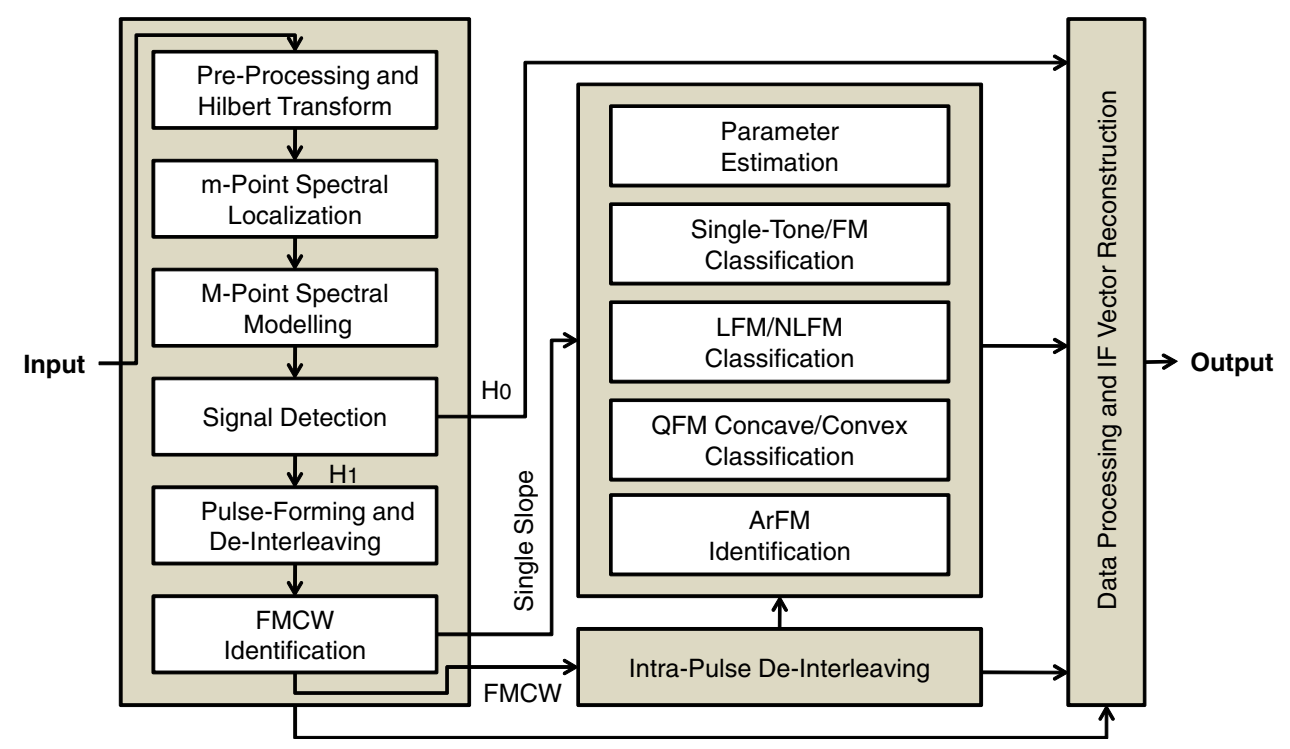

Figure 1. Analysis architecture for detection, estimation, classification and reconstruction of LPI radar signals. 
consolidation of signal energy, especially in handling large bandwidth signals under low SNR. Localization is designed as a search problem in a multi-dimensional, constrained, parametric space with the following assumptions and processing adaptations for dimension reduction:

- Received real signal is transformed into analytic signal by HT. This facilitates MFB-based complex projection analysis without estimating initial phase offset, $\varphi$. To show this, consider complex functions $\mathbf{x}$ and $\mathbf{y}$ with initial phase offsets $\varphi$ and zero, respectively. Projection of $\mathbf{y}$ onto $\mathbf{x}$ and computing complex magnitude results in

$$
\begin{aligned}
\left|\mathbf{y}^{H} \mathbf{x}\right| & =\left|\sum_{n}\left[\exp \left(-j \omega_{y} n\right)\right]\left[\exp \left(j\left\{\omega_{x} n+\varphi\right\}\right)\right]\right| \\
& =\left|\sum_{n}\left[\exp \left(j\left(\omega_{x}-\omega_{y}\right) n\right)\right]\right| .
\end{aligned}
$$

- Received $(L \times 1)$ vector is analysed piecewise in $(m \times$ 1) sub-frames, where $m \ll L$. IF is assumed to be approximately linear over $m$ samples. This takes away the need to estimate FM period in each sub-frame and facilitates initial localization of SOIs with continuoustime IF under piecewise-linearity assumption.

- Overlapped sub-frames are progressively formed by advancing by ' $l$ ' samples, where $l<m$. Each sub-frame has $l$ new samples and $(m-l)$ samples belonging to previous sub-frame. Sample reuse captures local TF signatures within sub-frames up to $l$-point resolution.

With reduced dimensions, a compact MFB is designed in the form of an under-determined sensing matrix, $\mathbf{A}_{m \times N}=\left[\begin{array}{llll}\mathbf{a}_{1} & \mathbf{a}_{2} & \ldots & \mathbf{a}_{N}\end{array}\right]$, with $m \ll N$. Each column of this matrix is a complex, unit-norm, noise-free, matching function, constructed under linearity and continuity assumptions, spanning the search space. Grid spacing is designed to bind sub-frame under analysis within the neighbourhood of true parameters. Signal sub-frames are projected onto the MFB progressively and columns showing highest projection magnitude provide spectral localization. Fine estimates are obtained from a dynamically built local search space, using initial estimates. This procedure is repeated over the length of observation vector to consolidate the underlying IF of SOI.

At this point, an important discussion is in order. IF sub-frames of SOI may be linear/non-linear and/or periodic/aperiodic. For linear cases, objective function formulation with linear model for descent algorithms and optimal, iterative, multi-signal recovery is possible as shown in [22]. However, for non linear cases, objective function formulation for direct recovery is not practically feasible. Consider a practical, multi-signal scenario with
SOI of highest amplitude being an NLFM. Iterative recovery in [22] gives the optimal linear estimate, in the first iteration. This linear structure is not the true definition of NLFM IF and regularization results in residue, leading to over/underestimation of number of atoms. The proposed method is designed to progressively acquire non-linear IF and reconstruct NLFM by least squares error minimization. Extension of the proposed method in line with the method of [22] is a potential solution for multi-signal analysis without constraint on linearity of IF. However, the same is beyond the scope of current work.

\section{4 m-Point spectral localization}

Next, formulation of spectral localization is presented. The received signal $\mathbf{b}_{L \times 1}$ has ' $N_{s}$ ' sub-frames to be analysed, where $N_{s}=\left\lfloor\frac{L-m}{l}\right\rfloor+1$. Progressive signal sub-frame projection onto MFB, $\mathbf{A}$, is analytically expressed as

$$
\mathbf{u}_{k, i}=\underset{i=1,2, \ldots, N}{\arg \max }\left|\mathbf{a}_{i}^{H} \mathbf{b}_{k}\right|, \quad k=1,2, \ldots, N_{s}
$$

where $\mathbf{b}_{k} \in R^{m \times 1}$ denotes $k^{\text {th }}$ subframe of $\mathbf{b}$, formed by progressively advancing by $l$-samples; $\mathbf{u}_{k, i}$ is the parametric vector index of $\mathbf{a}_{i}$ obtained as initial estimate for $\mathbf{b}_{k}$. This process is equivalent to progressive windowing of $\mathbf{b}$, i.e.

$$
\mathbf{b}_{k}=\{b[n]\}, \quad n \in[l(k-1)+1 \quad l(k-1)+m] .
$$

MFB binds sub-frames close to true parametric grids; however, estimates are coarse. MFB with finer grid resolution has the associated search complexity in multiple dimensions. $\mathbf{A}$ is designed with just enough grid resolution for reliable localization, and is driven by system design considerations. To obtain fine estimates using initial estimates, a local search space denoted by $\mathbf{D}_{m \times N_{i}}^{(i)}=\left[\begin{array}{llll}\mathbf{d}_{1}^{(i)} & \mathbf{d}_{2}^{(i)} & \ldots & \mathbf{d}_{N_{i}}^{(i)}\end{array}\right]$ is dynamically generated in the first neighbourhood of $\mathbf{u}_{k, i}$ and search for optimal column is carried out for each sub-frame as shown:

$$
\mathbf{u}_{k, i, j}=\underset{j=1,2, \ldots, N_{i}}{\arg \max }\left|\mathbf{d}_{j}^{(i)^{H}} \mathbf{b}_{k}\right|, \quad k=1,2, \ldots, N_{s}
$$

where $\mathbf{u}_{k, i, j}$ is the parametric vector index of $j^{\text {th }}$ column of $\mathbf{D}^{(i)}$ obtained as fine estimate for $\mathbf{b}_{k}$ based on $\mathbf{a}_{i}$.

\subsection{M-point spectral modelling}

Input to this step is $\left(N_{s} \times 1\right)$ IF estimate and the objective is to synthesize $M$-point IF frames by Taylor's series modelling. Consider the $i^{\text {th }}$ frame of SOI denoted by $\mathbf{b}_{M \times 1}^{(i)}$ and its IF estimate, $\hat{\mathbf{f}}_{\kappa \times 1}^{(i)}$, where $\kappa=\frac{M}{l}+1$. A frame is spectrally represented by $\kappa$ equidistant IF points separated by $l$ 
samples; $\hat{\mathbf{f}}^{(i)}$ is approximated by second order Taylor's series as shown [27]:

$$
\left[\begin{array}{c}
\hat{v}_{1}^{(i)} \\
\hat{v}_{2}^{(i)} \\
\hat{v}_{3}^{(i)}
\end{array}\right]=\underset{\left\{v_{1}^{(i)}, v_{2}^{(i)}, v_{3}^{(i)}\right\}}{\arg \min } \sum_{n}\left[\hat{f}^{(i)}[n]-\left(v_{1}^{(i)} n^{2}+v_{2}^{(i)} n+v_{3}^{(i)}\right)\right]^{2}
$$

$$
\left[\begin{array}{c}
\hat{v}_{1}^{(i)} \\
\hat{v}_{2}^{(i)} \\
\hat{v}_{3}^{(i)}
\end{array}\right]=\left[\begin{array}{ccc}
\sum_{n} n^{4} & \sum_{n} n^{3} & \sum_{n} n^{2} \\
\sum_{n}^{n} n^{3} & \sum_{n}^{n} n^{2} & \sum_{n}^{n} n \\
\sum_{n}^{n} n^{2} & \sum_{n}^{n} n & \kappa
\end{array}\right]^{-1}\left[\begin{array}{c}
\sum_{n} n^{2} \hat{f}^{(i)}[n] \\
\sum_{n} n \hat{f}^{(i)}[n] \\
\sum_{n} \hat{f}^{(i)}[n]
\end{array}\right]
$$

where all summations are over $n=0, l, \ldots, M$. Recognizing that parametric vector $\boldsymbol{v}^{(i)}$ closely defines the SOI slope, underlying phase difference vector, $\hat{\dot{\boldsymbol{\Phi}}}^{(i)}$, is constructed using

$$
\hat{\dot{\Phi}}^{(i)}=\hat{v}_{1}^{(i)} n^{2}+\hat{v}_{2}^{(i)} n+\hat{v}_{3}^{(i)}, \quad n=0, l, \ldots, M .
$$

The corresponding phase vector $\hat{\Phi}^{(i)}$ is computed as

$$
\begin{aligned}
\hat{\boldsymbol{\Phi}}^{(i)} & =\frac{\hat{v}_{1}^{(i)} n^{3}}{3}+\frac{\hat{v}_{2}^{(i)} n^{2}}{2}+\hat{v}_{3}^{(i)} n+\varphi \\
& =\hat{\omega}_{1} n^{3}+\hat{\omega}_{2} n^{2}+\hat{\omega}_{3} n+\varphi, \quad n=1,2, \ldots, M .
\end{aligned}
$$

where $\varphi$ is random phase offset uniformly distributed in the range $\left[\begin{array}{ll}0 & 2 \pi\end{array}\right]$ radians. $\left\{\hat{\omega}_{1}, \hat{\omega}_{2}, \hat{\omega}_{3}\right\} \triangleq \hat{\Omega}$ are optimization coefficients of Eq. (12). Corresponding complex, synthesized, ith frame of $\mathbf{b}$, denoted by $\check{\mathbf{b}}^{(i)}$, is computed as follows:

$$
\check{\mathbf{b}}^{(i)}=\alpha^{(i)}\left[\cos \left(2 \pi \Phi^{(i)}\right)+j \sin \left(2 \pi \Phi^{(i)}\right)\right] .
$$

$\check{\mathbf{b}}^{(i)}$ is a close approximation of $\mathbf{b}^{(i)}$ except for the unknown $\varphi$ in $\hat{\boldsymbol{\Phi}}^{(i)} ; \alpha^{(i)}$ is mean magnitude of $i^{\text {th }}$ frame. Next step is to phase-synchronize $\check{\mathbf{b}}^{(i)}$ with $\mathbf{b}^{(i)}$ using the formulation

$$
\hat{\varphi}^{(i)}=\underset{0 \leq \varphi \leq 2 \pi}{\arg \min }\left\|\mathbf{b}^{(i)}-\check{\mathbf{b}}^{(i)}(\varphi)\right\|^{2} .
$$

Using $\hat{\varphi}^{(i)}$, we get $\hat{\mathbf{b}}^{(i)}$, close to $\mathbf{b}^{(i)}$ in magnitude, frequency, phase and IF. Here, there are three possibilities, viz.

- Case-1: $\mathbf{b}^{(i)}$ contains noise only in all $M$ samples,

- Case-2: $\mathbf{b}^{(i)}$ contains signal+noise in all $M$ samples,

- Case-3: $\mathbf{b}^{(i)}$ is partly noise only and partly signal+ noise.
If Case-1 is true, under AWGN, $\hat{\mathbf{\Phi}}^{(i)}$ is a random distribution of frequencies over DRx bandwidth. Taylor's series approximation still constructs $M$-point, smooth and continuous slope. If Case-2 is true, $\hat{\mathbf{b}}^{(i)}$ is a close reconstruction of $\mathbf{b}^{(i)}$ in magnitude, angle and initial phase offset. If Case- 3 is true, Taylor's series reconstruction is at best a poor approximation and at worst a large deviation from true IF. This procedure of piecewise IF reconstruction is done over the entire length of observation vector without any frame overlap.

\subsection{IC-based signal detection}

The objective of this stage is signal detection in M-sample frames. Detection in the received noisy process is performed by hypotheses testing under minimum description length (MDL) criterion and complex, Gaussian, vector PDF assumption $[14,15]$. Defining MDL IC for the $q^{\text {th }}$ order distribution by the relation

$$
I C_{q}=-\log _{e}\left[p\left(\mathbf{b} ; \hat{\Theta}^{(q)}\right)\right]+\text { penalty term. }
$$

The first term on RHS is negative log-likelihood function of the complex, Gaussian PDF, $p\left(\mathbf{b} ; \hat{\Theta}^{(q)}\right)$. The second term is a penalty function, which depends on analysis vector length, model order and SOI structure. Before estimation, IC is computed using PDF of the analytic signal, $p\left(\mathbf{b} ; \hat{\Theta}^{(0)}\right)$. After parametric estimation, IC is computed using PDF of the residue, $\left[\mathbf{b}^{(i)}-\hat{\mathbf{s}}^{(i)}\right]$, i.e., $p\left(\mathbf{b} ; \hat{\Theta}^{(1)}\right)$. Since, $\hat{\mathbf{s}}^{(i)}$ is constructed to be deterministic by 2 nd order Taylor's series, it is a poor reconstruction of noise and close estimate of SOI frame. If $\hat{\mathbf{s}}^{(i)}$ is indeed a good reconstruction of $\mathbf{s}^{(i)}$, IC cost function magnitude decreases, indicating signal detection. This process is repeated over the observation vector length, without sample overlap to detect individual frames and to obtain coarse PW estimate, up to $M$-point resolution.

\subsection{Pulse forming and deinterleaving}

In this step, signal detection within frames is carried out. When the noise vector is projected onto MFB, resultant magnitude is a function of noise projections. When signal+noise is projected, projection magnitude is a function of correlation between two signals and correlation between noise and match function. The noise process in both cases is identically distributed. Transition threshold is set as the average of mean projection magnitudes of noise only and signal+noise regimes. PW of each signal is estimated and deinterleaving is carried out by forming distinct slope vectors. Analysis in this stage is heavily dominated by noise. 


\subsection{FMCW identification and intra-pulse de- interleaving}

Pulses formed in the previous stage are tested for periodicity in IF. The objective of this step is to separate out FMCW signals, decompose them into multiple, unidirectional IF segments and analyse each segment independently. Segment-wise processing facilitates analysis of complex FMCW structures like up and down slopes designed as different types with different sub-periods. For each FMCW pulse, number of mean-crossing events and polarity of crossing are tabulated. Unidirectional IFs have one up or down crossing, whereas FMCW would have more than one crossing. Extrema points of the IF vector between two level crossings are computed. With the knowledge of all extrema points in the given IF, individual segments with unidirectional IF structures are separated out.

\subsection{Parameter estimation}

The objective of this step is estimation of intra-pulse modulation parameters. Inputs are number of distinct pulses detected, IF and complex magnitude vectors for each pulse/sub-pulse. Let $\mathbf{g}_{k}$ represent IF estimate of the $k^{\text {th }}$ detected pulse. Parameter estimation is carried out individually for each pulse by approximating corresponding IF vectors using second order Taylor's series model, $\hat{g}_{k}[n]=\hat{u}_{1}^{(k)} n^{2}+\hat{u}_{2}^{(k)} n+\hat{u}_{3}^{(k)}$. The chosen model closely approximates Unmod, LFM and QFM and is the basic representation for all continuous-time IF structures. The linear model is sub-optimal in approximating non-linear and arbitrary IF. Higher order Taylor's series models approximate arbitrary IF with better accuracy, but are computationally complex. The proposed cost function for parameter estimation is

$$
\left[\begin{array}{c}
\hat{u}_{1}^{(k)} \\
\hat{u}_{2}^{(k)} \\
\hat{u}_{3}^{(k)}
\end{array}\right]=\underset{u_{1}^{(k)}, u_{2}^{(k)}, u_{3}^{(k)}}{\arg \min } \sum_{n}\left[g_{k}[n]-\left(u_{1}^{(k)} n^{2}+u_{2}^{(k)} n+u_{3}^{(k)}\right)\right]^{2}
$$

where range of summation is $\left[\begin{array}{ll}0 & M_{k}\end{array}\right]$ in steps of $l$-samples and $M_{k}$ is the PW of $k^{\text {th }}$ pulse. The solution is obtained in closed form as in Eq. (18) and $\hat{\mathbf{g}}_{k}$ is reconstructed using coefficient vector, $\hat{\mathbf{u}}^{(k)}$. Estimates of centre frequency, modulation bandwidth and modulation period are computed using

$$
\begin{gathered}
\hat{F}_{k}=\hat{g}_{k}[0]+0.5\left(\hat{g}_{k}\left[M_{k}\right]-\hat{g}_{k}[0]\right) \\
\hat{B}_{k}=\left|\hat{g}_{k}\left[M_{k}\right]-\hat{g}_{k}[0]\right| \\
\hat{T}_{k}=\left(x_{k}[2]-x_{k}[1]\right) T_{s}
\end{gathered}
$$

where $\mathbf{x}_{k}$ gives start and end sample indices of the $k^{\text {th }}$ pulse and $T_{s}$ denotes sampling period. If SOI is FMCW, up/down slopes are identified and intra-pulse deinterleaving is done before estimation. Each slope is independently modelled and results are fused to give the final estimates for each pulse.

\subsection{Modulation classification}

Modulation classification is done for identifying candidate SOIs, viz., Unmod, LFM, QFM (concave) and QFM (convex). Modulated SOIs may have either positive or negative slope. A linear programming approach [27] using polynomial models is followed. SOI is declared modulated if $\hat{B} \geq B_{t h}$, where $B_{t h}$ is bandwidth threshold, and analysis proceeds further, else processing terminates with class declaration as 'Unmod'.

5.10a Linear/Non-Linear FM Classification: This step is a test of linearity of IF structure of SOI. Distinction is sought between purely linear and purely non-linear slopes by the following cost function formulation, minimizing SSE of error vector to identify the matching model, i.e.,

$$
\begin{gathered}
\mathcal{C}_{\mathrm{L}}=\sum_{n}\left[g_{k}[n]-\left(u_{1}^{(k)} n+u_{2}^{(k)}\right)\right]^{2} \\
\mathcal{C}_{\mathrm{NL}}=\sum_{n}\left[g_{k}[n]-\left(u_{1}^{(k)} n^{3}+u_{2}^{(k)} n^{2}+u_{4}^{(k)}\right)\right]^{2}
\end{gathered}
$$

where $n \in\left[\begin{array}{ll}0 & M_{k}\end{array}\right]$ in steps of $l$-samples. Solutions minimizing $\mathscr{C}_{\mathrm{L}}$ and $\mathscr{C}_{\mathrm{NL}}$ are obtained in closed form as shown in Eq.(18). For LFM signal, $\mathscr{C}_{L} \leq \mathscr{C}_{N L}$. If the linear model is chosen, the process stops with class declaration as 'LFM'.

5.10b Quadratic (Concave/Convex) FM Classification: This step is to chose QFM distinctly among non-linear models and to further classify based on up/down and concave/convex shape. Cost function for quadratic models (concave up slope and convex down slope) is given by

$$
\mathscr{C}_{\mathrm{Q} 1}=\sum_{n}\left[g_{k}[n]-\left(u_{1}^{(k)} n^{2}+u_{3}^{(k)}\right)\right]^{2}
$$

where $n \in\left[\begin{array}{ll}0 & M_{k}\end{array}\right]$ in steps of $l$-samples. Cost function $\mathscr{C}_{\mathrm{Q} 2}$ for quadratic convex up and concave down slope models are computed as before with time base being negative and flipped. Solutions are obtained by solving for unknowns as shown in Eq. (18). The model with minimum cost function magnitude is chosen as the likely solution.

5.10c Arbitrary FM Identificaton: Pearson's correlation coefficient between IF and its second order Taylor's series model is used as the metric to identify ArFM. Here, the objective is to sense loss of correlation in case SOI is not LFM/QFM, set ArFM flag and reconstruct SOI using fourth-order Taylor's series. But in many cases, even 
fourth-order Taylor's series may not be a suitable model, resulting in large deviation in slope reconstruction. This step aims at identifying SOIs without pre-defined analytical models and facilitate their later inclusion with appropriate model definition.

\subsection{Data processing and IF vector reconstruction}

The function of this stage is consolidation and presentation of results obtained in various stages of analysis. If hypothesis $H_{0}$ holds, noise vector and initial localization are presented as output. Under $H_{1}$, detection, estimation and classification results are collated to form a pulse descriptor matrix along with three estimate vectors, viz., TF localization, Taylor series frame synthesis and IF of SOI.

\section{Performance analysis}

The proposed method offers design flexibility to meet specific objectives with allied constraints. MFBs with longer vectors offer good detection performance. As subframe length increases, piecewise linearity assumption for non-linear slopes gets progressively violated and hence approximation accuracy decreases. Shorter match functions more closely approximate arbitrary slopes within sub-frames with good estimation accuracies and smaller MFBs. The penalty is detection performance for lower SNRs. Overall performance also depends on parametric grid configuration, with denser grids being robust against noise. The proposed method has been evaluated with two MFBs $\mathbf{A}_{1}^{\left(1024 \times N_{1}\right)}$ and $\mathbf{A}_{2}^{\left(512 \times N_{2}\right)}$ designed for good detection performance at low SNRs and lower computation complexity, respectively. Following parametric space, under-band-pass sampling scheme is used for test signal generation and analysis. Signal classes considered are unmod, LFM, QFM (concave) and QFM (convex). Analysis for null hypothesis is carried out with real AWGN as input.

- Centre frequency $(F)$ : $100-600 \mathrm{MHz}, \quad 1 \mathrm{kHz}$. resolution.

- FM bandwidth (B): 2-500 MHz, $1 \mathrm{kHz}$. resolution.

- Initial phase offset $(\varphi)$ : $0-359^{\circ}$ in unit steps.

- FM period (T): 3-60 $\mu$ s in unit sample steps.

- Receiver noise: real AWGN, $\mathbf{w}_{r} \sim \mathcal{N}\left(\mathbf{0}, \frac{\sigma^{2}}{2} \mathbf{I}_{\mathbf{L}}\right)$.

- Input SNR: minimum $-15 \mathrm{~dB}$, maximum $15 \mathrm{~dB}$.

\subsection{Detection performance}

Detection test set-up involves random, synthetic SOI generation within parametric space and analysis using the proposed method; 1000 trials were carried out for each
SNR case, separately for two MFBs and results are presented in figure 2 .

In an $(L \times 1)$ observation vector, $(T \times 1)$ signal appears in multiple $(M \times 1)$ frames with possibility of two end frames with partial signal presence. Number of frames, $N_{f}$, with complete signal presence is computed and the pulse is considered detected only if all $N_{f}$ frames are detected. As SNR decreases, noise dominates signal correlation peaks, resulting in random spectral localization. Accuracy of signal synthesis for applying IC criterion decreases, resulting in some of the frames missing detection. Signals with parametric values lying farthest from the MFB grid points are the most vulnerable. By decreasing parametric resolution, probability of detection $\left(P_{d}\right)$ improves in transition region at increased computation complexity as shown in figure 2 for MFBs, $\mathbf{A}_{1}$ and $\mathbf{A}_{2}$. Cut-off SNR with $P_{d}>0.99$ for $\mathbf{A}_{1}$ and $\mathbf{A}_{2}$ are -10 and $-8 \mathrm{~dB}$, respectively, with graceful degradation over $6 \mathrm{~dB}$ down the range. The $2 \mathrm{~dB}$ SNR advantage of $\mathbf{A}_{1}$ over $\mathbf{A}_{2}$ comes at approximately doubled computation. Above $-8 \mathrm{~dB} \mathrm{SNR}$, both MFBs have similar $P_{d}$ performance. In the current performance quantification frame-work, even a single undetected frame would render the entire pulse undetected. Practical SOIs have pulses comprising tens to hundreds of frames, depending on sampling frequency and PW. $P_{d}$ performance under such stringent criterion brings out detection robustness of the MDL IC method adopted.

\subsection{Probability of false alarm}

Consider a DRx with $500 \mathrm{MHz}$ bandwidth. The Received process under hypothesis $H_{0}$ is assumed to be real WGN at $-75 \mathrm{dBm}$ power level, as nominally observed in practical receivers of that class. One detection decision is made on each frame based on IC cost function thresholding. Each trial in probability of false alarm $\left(P_{f a}\right)$ performance analysis corresponds to a frame detection. Observation vector (WGN, $\left.\mathbf{b}_{\mathbf{r}}=\mathbf{w}_{r}\right)$ of length $\left(M \times 10^{5}\right)$ is input for the simulations conducted using $\mathrm{MFBs}, \mathbf{A}_{1}$ and $\mathbf{A}_{2}$ with $10^{5}$

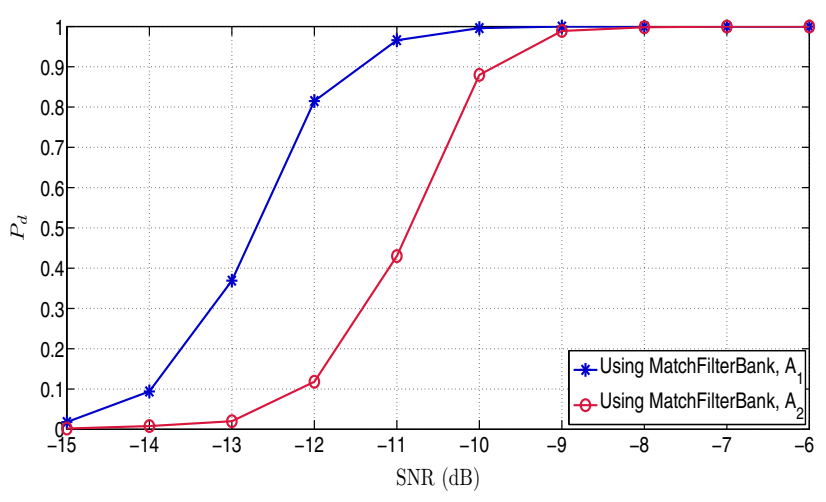

Figure 2. MDL-IC based signal detection performance using MFBs, $\mathbf{A}_{1}^{\left(1024 \times N_{1}\right)} \& \mathbf{A}_{2}^{\left(512 \times N_{2}\right)}$ in a $500 \mathrm{MHz}$ bandwidth DRx. 
trials in each case. No false alarms were reported in either case. In the proposed method, missed detection of a frame in the midst of a pulse is far more critical than false detection of frames. Practically, such frames do not qualify as valid SOIs, due to their narrow PW and lack of structured occurrences. For the current test set-up, MDL IC thresholding and $P_{d}$ performance as in figure 2, the proposed solution is shown to have $P_{f a} \leq 10^{-5}$.

\subsection{Estimation performance}

Estimation performance is quantified in terms of mean and standard deviation of parametric estimation errors. Simulation results for centre frequency, modulation bandwidth and modulation period are presented in figures 3-5. Estimation error mean curves are observed to be asymptotically tending to zero over SNR, whereas standard deviation curves are observed to reach convergence values over SNR. Accuracy depends on fine localization before detection and model approximation after detection. Fine localization resolutions for $F$ and $B$ are set to 0.1 and $1 \mathrm{MHz}$, respectively. Convergence $\sigma_{\tilde{F}}$ and $\sigma_{\tilde{B}}$ are on the order of tens of
$\mathrm{kHz}$ as shown, respectively, in figures $3 \mathrm{~b}$ and $4 \mathrm{~b}$. Convergence $\sigma_{\tilde{T}}$ is approximately $0.11 \mu \mathrm{s}$. Better performance of $\mathbf{A}_{2}$ highlights fine capturing of $\mathrm{TF}$ signatures by shorter match functions. However, estimation is done post-detection, where $\mathbf{A}_{1}$ has better $P_{d}$. Below cut-off SNRs, although signal is detected, estimates have large bias and variance. The proposed method is shown to be statistically consistent with minimum variance, unbiased estimator behaviour asymptotically and graceful degradation over SNR.

\subsection{Classification performance}

For each SOI class, 1000 trials were conducted over the randomized parametric space at $-6 \mathrm{~dB}$ SNR. $\hat{B}_{t h}$ is set at $2 \mathrm{MHz}$ for modulation detection. Performance, quantified in terms of confusion matrices, is presented in figure $6 a$ and $b$ for $\mathbf{A}_{1}$ and $\mathbf{A}_{2}$, respectively. Classification between unmodulated/modulated and linear/non-linear cases is seen to be robust, while higher confusion probability is seen in case of LFM signals. Frame-length-dependent distinction is also seen, with $\mathbf{A}_{1}$ showing better classification than $\mathbf{A}_{2}$. Above $-6 \mathrm{~dB}$ SNR, both matrices are close to identity. For

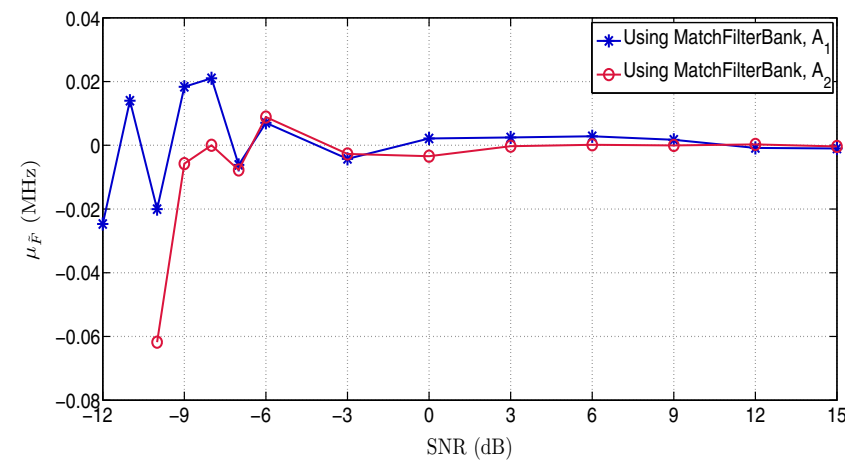

(a)

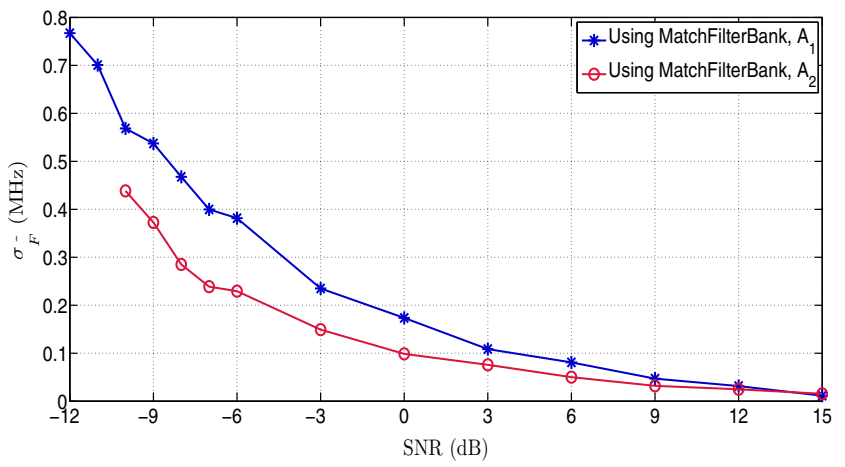

(b)

Figure 3. Centre-frequency estimation performance for $100 \mathrm{MHz} \leq \mathrm{F}_{\mathrm{n}} \leq 600 \mathrm{MHz}$, over 1000 trials, in a 500-MHz DRx. (a) Centrefrequency estimation error mean. (b) Centre-frequency estimation error sigma.

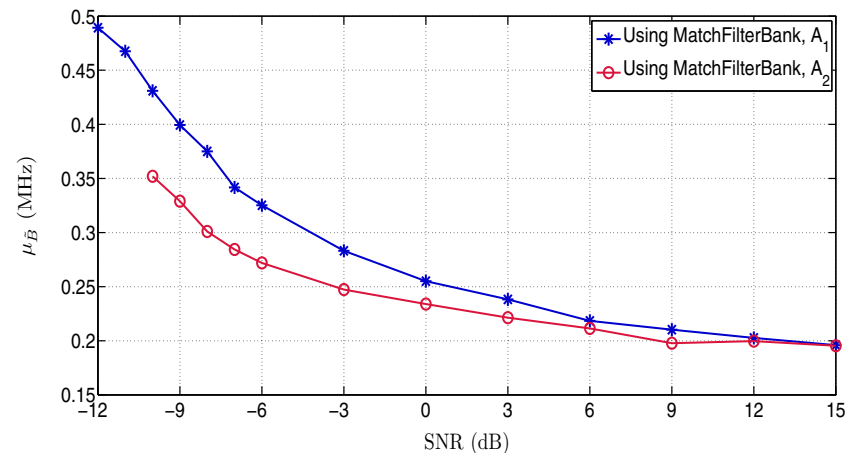

(a)

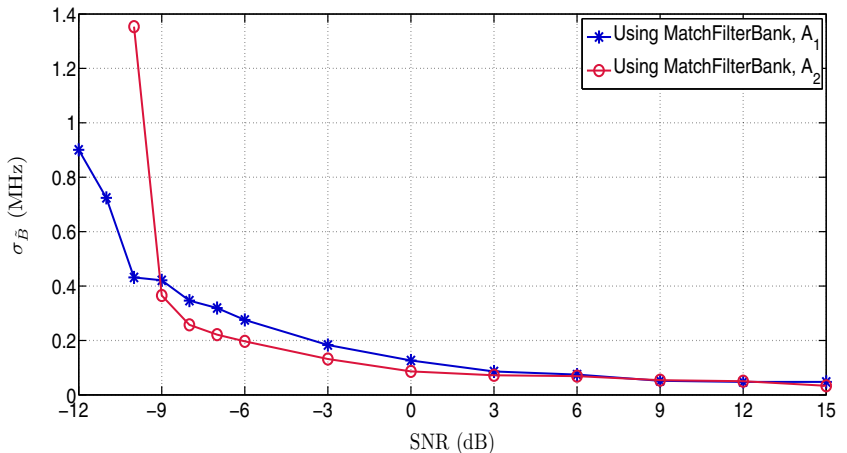

(b)

Figure 4. FM bandwidth estimation performance for $2 \mathrm{MHz} \leq \mathrm{B}_{\mathrm{n}} \leq 500 \mathrm{MHz}$, over 1000 trials, in a 500-MHz DRx. (a) Modulationbandwidth estimation error mean. (b) Modulation-bandwidth estimation error sigma. 


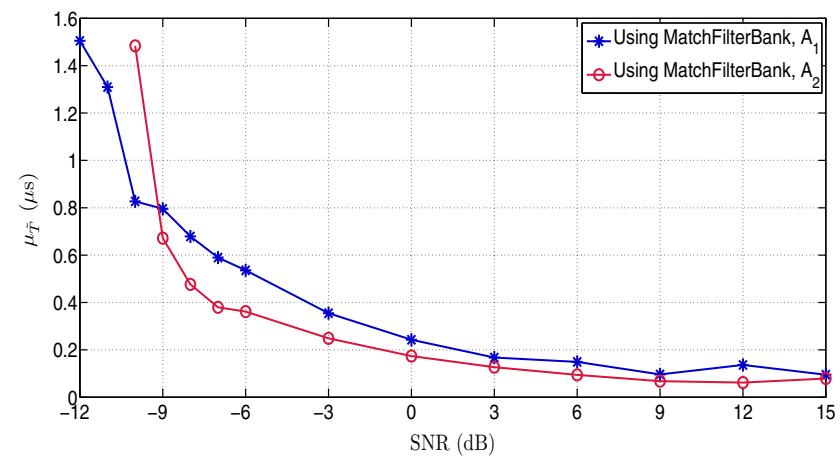

(a)

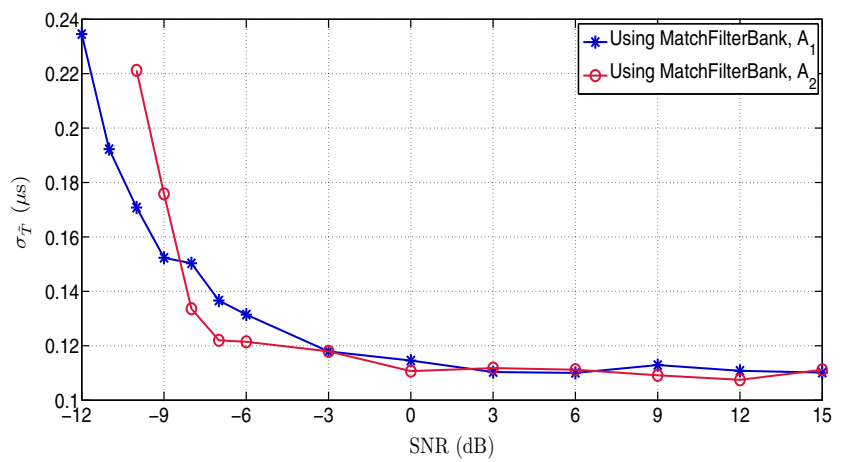

(b)

Figure 5. FM period estimation performance for $3 \mu \mathrm{s} \leq T_{n} \leq 60 \mu \mathrm{s}$, over 1000 trials, in a 500-MHz DRx. (a) Modulation-period estimation error mean. (b) Modulation-period estimation error sigma.

\begin{tabular}{|c|c|c|c|c|}
\hline & UNMOD & LFM & $\begin{array}{c}\text { QFM } \\
\text { (concave) }\end{array}$ & $\begin{array}{c}\text { QFM } \\
\text { (convex) }\end{array}$ \\
\hline UNMOD & 1 & 0 & 0 & 0 \\
\hline LFM & 0.001 & 0.998 & 0 & 0.001 \\
\hline $\begin{array}{c}\text { QFM } \\
\text { (concave) }\end{array}$ & 0 & 0.001 & 0.999 & 0 \\
\hline $\begin{array}{c}\text { QFM } \\
\text { (convex) }\end{array}$ & 0.002 & 0 & 0 & 0.998 \\
\hline
\end{tabular}

(a)

\begin{tabular}{|c|c|c|c|c|}
\hline & UNMOD & LFM & $\begin{array}{c}\text { QFM } \\
\text { (concave) }\end{array}$ & $\begin{array}{c}\text { QFM } \\
\text { (convex) }\end{array}$ \\
\hline UNMOD & 1 & 0 & 0 & 0 \\
\hline LFM & 0.003 & 0.995 & 0.001 & 0.001 \\
\hline $\begin{array}{c}\text { QFM } \\
\text { (concave) }\end{array}$ & 0.001 & 0.003 & 0.996 & 0 \\
\hline $\begin{array}{c}\text { QFM } \\
\text { (convex) }\end{array}$ & 0.001 & 0.003 & 0 & 0.996 \\
\hline
\end{tabular}

(b)

Figure 6. Classification performance for four SOI classes, i.e., unmodulated, linear FM, quadratic concave FM and quadratic convex FM at $-6 \mathrm{~dB}$ SNR, using $\mathbf{A}_{1}$ and $\mathbf{A}_{2}$, in a 500-MHz bandwidth digital receiver, with 1000 trials for each SOI case. (a) Confusion matrix for MFB, $\mathbf{A}_{1}^{\left(1024 \times N_{1}\right)}$. (b) Confusion matrix for MFB, $\mathbf{A}_{2}^{\left(512 \times N_{2}\right)}$.

lower SNRs, noise-dependent graceful degradation is observed.

\section{LPI radar scenario simulation}

In this section, performance of the proposed method for realistic EW scenarios is demonstrated in MATLAB $^{\circledR}$. Signal, noise and modulation parameters are discussed in practical units (i.e., expressing time in $\mu$ s, frequency in $\mathrm{MHz}$, etc.) instead of normalized units, as conventionally done, in order to give practical appreciation. Scenario simulation demonstrates analysis behaviour under constraints like

- Static behaviour under no signal condition,

- Processing unmodulated signals,

- Capability to process SOIs with narrow/long PW, large/small FM slopes and wide/narrow FM bandwidth,
- Handling highly constrained scenarios like large slope and wide bandwidth, very narrow slope and very large modulation period, occurrence of both linear and nonlinear slopes within a pulse, etc. and

- Capability to process periodic and arbitrary IF structures.

Figure 7 a shows analysis of receiver noise without SOI, resulting in random spectral localization over DRx bandwidth. Figure $7 \mathrm{~b}-\mathrm{e}$ demonstrates processing of basic SOIs, viz., unmod, LFM and NLFM. Figure $7 \mathrm{~b}$ shows linear modelling of unmodulated signal at different stages of analysis. Tightly bound localization around the true frequency and ability to tolerate outliers are shown. LFM in figure $7 \mathrm{c}$ has a slope of $20 \frac{\mathrm{MHz}}{\mu \mathrm{s}}$ and sweeps the entire DRx bandwidth, showing performance under large-slope and wide-bandwidth conditions. In figure $7 \mathrm{~d}$, nature of convergence by SSE minimization using finite resolution estimates is shown for QFM concave signal with just $0.03 \frac{\mathrm{MHz}}{\mu \mathrm{s}}$ slope. Performance under small-slope, narrow- 
bandwidth and long-PW constraints is demonstrated here. Figure $7 \mathrm{e}$ is an example of generic NLFM with nominal parametric values. Figure $7 \mathrm{f}$ demonstrates processing of broad band, large TBP LFMCW, a widely used candidate for LPI signal design. In figure $7 \mathrm{~g}$, a special case of FMCW with linear up slope and quadratic down slope and unequal modulation periods for up and down slopes is shown. This example demonstrates adaptive processing of various FMCW structures. Figure $7 \mathrm{~h}$ is a simulated test case of ArFM type, with non-linear slope followed by linear slope. Initial localization and frame approximation performance is the same as in basic SOI cases. However, in the absence of specific model, final reconstruction is approximate, as modelled by fourth order Taylor's series, with an indication of ArFM flag. This example demonstrates adaptive processing capability without any prior information on incoming signal structure. In all these examples, it may be observed that TF localization and Taylor's series frame synthesis curve closely approximates the true IF of SOIs.

\section{Considerations in selection of methods}

As elaborated in literature survey, various methods exist to address the current problem. Considerations for selecting specific methods for the proposed solution and the corresponding advantages are enumerated here.

- The proposed MFB method is a parallel implementation, much simpler than spectrogram, WVD, PWVD, CWD and wavelets, is free of cross terms and is independent of FM class of SOI. Choice of MFB is also due to its suitability for implementation in DRx firmware/software.

- MDL IC based on complex, Gaussian PDF is a coherent method with robust detection and has flexibility of trade-off between $P_{d}$ and $P_{f a}$ by controlling penalty term. Thus, it is a natural choice for current solution.

- Novelty of the proposed solution, in comparison with other solutions, is SOI detection based on Taylor's series modelling. This approach imparts capability to address the entire family of FM signals, which is of practical significance in real-life radar threat scenarios.

- FM signals, by design, have continuous IF, at least over short durations. Polynomial approximation using higher order Taylors series offers optimal solution for continuous structures, and hence, it is chosen for estimation and classification. It is simpler than image processing methods and has better SNR performance.

\section{Performance comparison}

The proposed solution is compared with regard to overall performance with methods in [13, 16, 19, 22, 23].
- In [13], referring to section 5, $P_{d}=0.9$ and $P_{f a}>0.01$ have been reported for $-15 \mathrm{dBSNR}$ for detection and estimation of LFMCW using PWVHT. The method caters to multi-signal analysis, with demonstrated performance for LFM/LFMCW.

- The proposed approach has similarities in [16], in that both solutions are coherent, acquire IF of the SOI and classification/estimation are on similar lines. [16] also focuses on time of arrival and emitter geo-location. Demonstrated performance for LFMCW is for SNR = $-4 \mathrm{~dB}$ and $P_{f a}=10^{-9}$ using short time harmonic modelling, clustering, association by KolmogorovSmirnov test and estimation by optimization.

- Both [19] and the proposed method identify that, currently, SNR range of -15 to $-10 \mathrm{~dB}$ is the current cut-off SNR region for LPI regime in EW systems. In [19], detection performance for SNR $>=-14 \mathrm{~dB}$ and $P_{f a}=10^{-4}$ is demonstrated for LFMCW signals.

- Refer to section 6 in [22]. For 1024 samples frame, detection sensitivity for $P_{d}=0.9$ and $P_{f a}=10^{-6}$ is in -1 to $1 \mathrm{dBSNR}$ range for linear chirplets considered. Multi-signal detection and estimation performance is demonstrated for linear chirplets class.

- Refer to section 6 in [23]. LFMCW detection sensitivity for 1024 samples frame, $P_{d}=0.9, P_{f a}=10^{-6}$, with $\frac{100 \mathrm{MHz}}{1 \mathrm{~ms}}$ and $\frac{500 \mathrm{MHz}}{1 \mathrm{~ms}}$ slopes is $-11 \mathrm{dBSNR}$. For SOIs with $\frac{1 \mathrm{MHz}}{1 \mu \mathrm{s}}, \frac{2 \mathrm{MHz}}{2 \mu \mathrm{s}}$ and $\frac{-40 \mathrm{MHz}}{4 \mu \mathrm{s}} \mathrm{FM}$ slopes, reported detection sensitivities are $-4,-6$ and $-11 \mathrm{~dB}$ SNRs respectively.

Performance summary of the proposed method is as follows:

- Detection, estimation and classification of periodic/ aperiodic SOIs with continuous-time IF using a single MFB;

- Detection sensitivity for $P_{d}=0.966, P_{f a}=10^{-5}$ is $-11 \mathrm{~dB}$ SNR with statistically consistent estimation;

- Confusion matrix close to identity for $\mathrm{SNR} \geq-6 \mathrm{~dB}$;

- Demonstrated performance in the parametric space, $2 \leq B(\mathrm{MHz}) \leq 500, \quad 3 \leq \mathrm{PW}(\mu \mathrm{s}) \leq 60 \quad$ and $0 \leq \frac{B}{T}\left(\frac{\mathrm{MHz}}{\mu \mathrm{s}}\right) \leq 20$; analysis is independent of initial phase offset;

- Provision to add new SOI classes, extension/reduction of parametric space and flexibility in MFB design and

- Proposed method is designed for single signal analysis and can process multiple interleaved SOIs.

The proposed method has detection and estimation performance comparable to those of the referred methods for LFM/LFMCW. These methods cater to multi-signal analysis, but are restricted to linear chirplet, LFM and LFMCW classes only. Strength of the proposed method lies in its capabilty to handle complex EW scenarios with unmodulated pulsed/CW, LFM/NLFM, linear/non-linear FMCW 


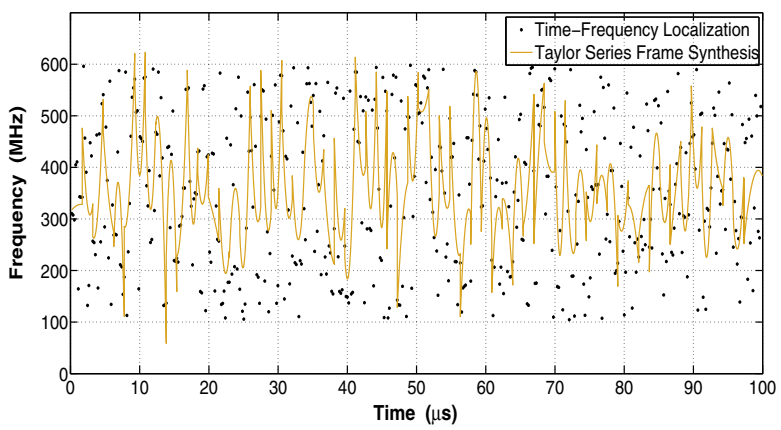

(a)

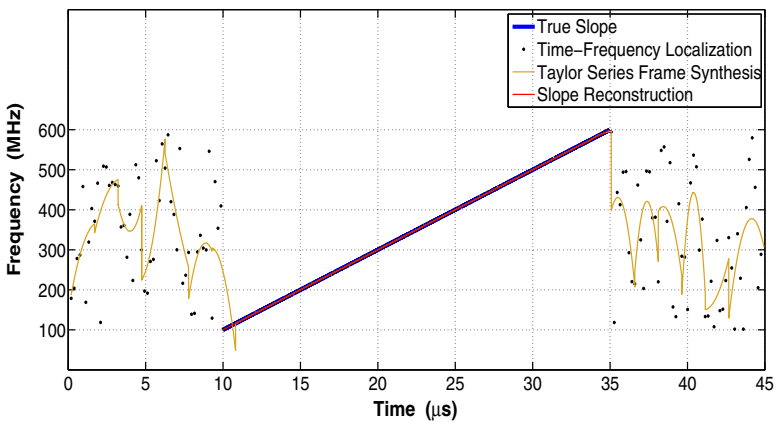

(c)

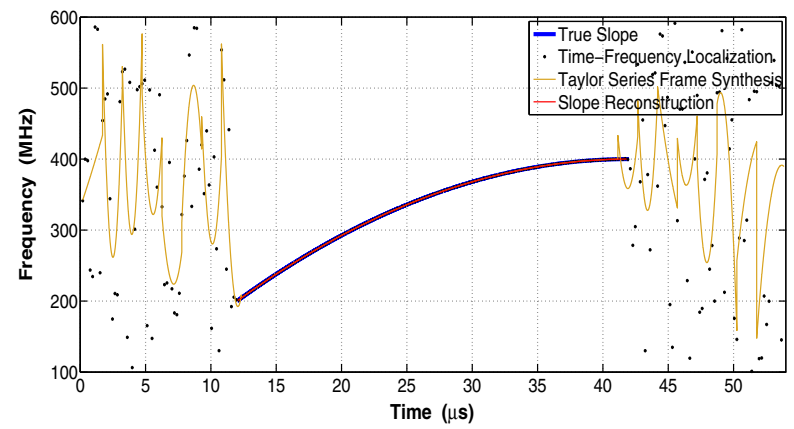

(e)

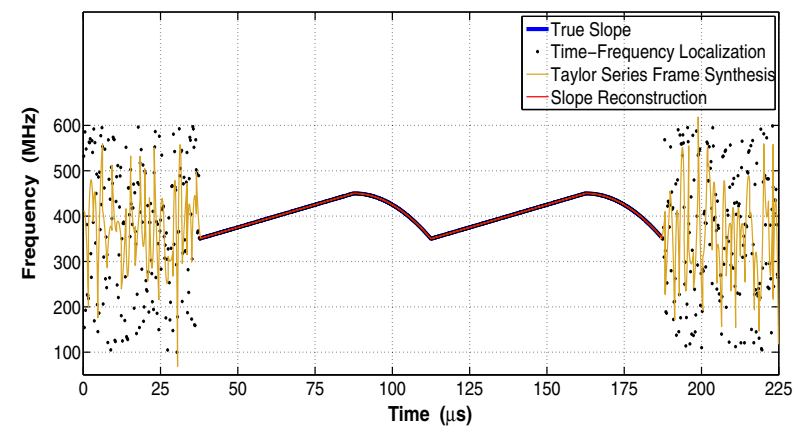

(g)

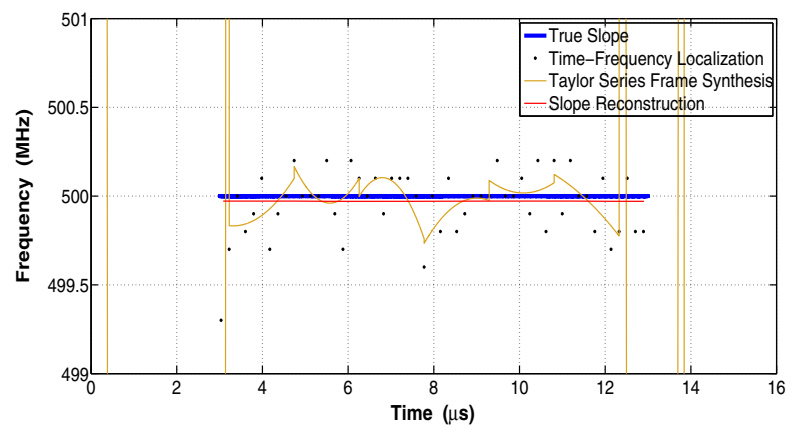

(b)

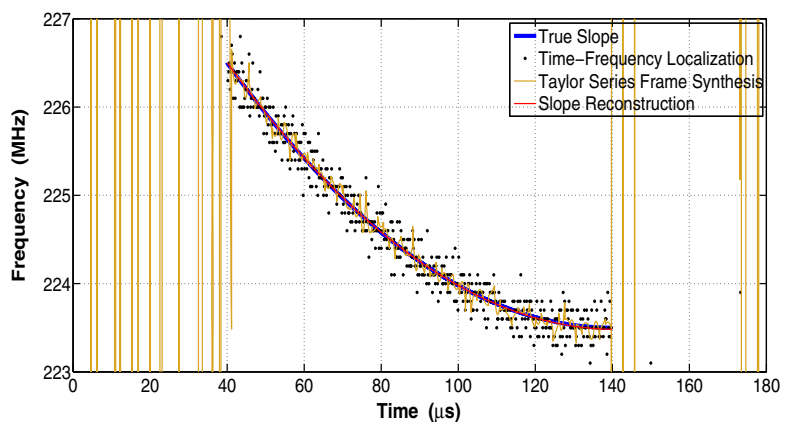

(d)

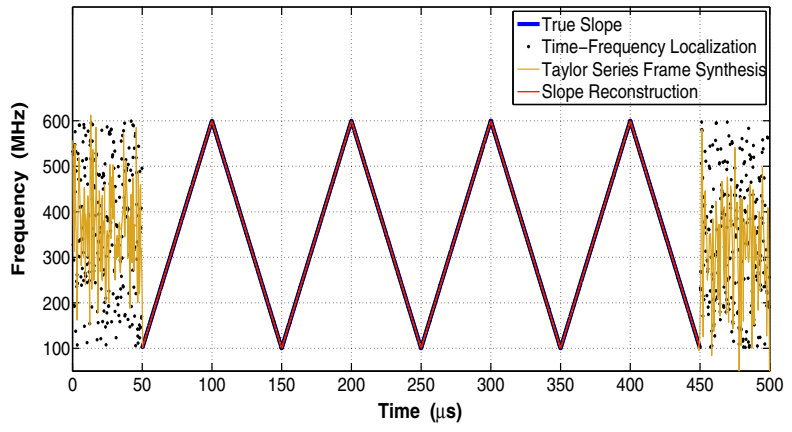

(f)

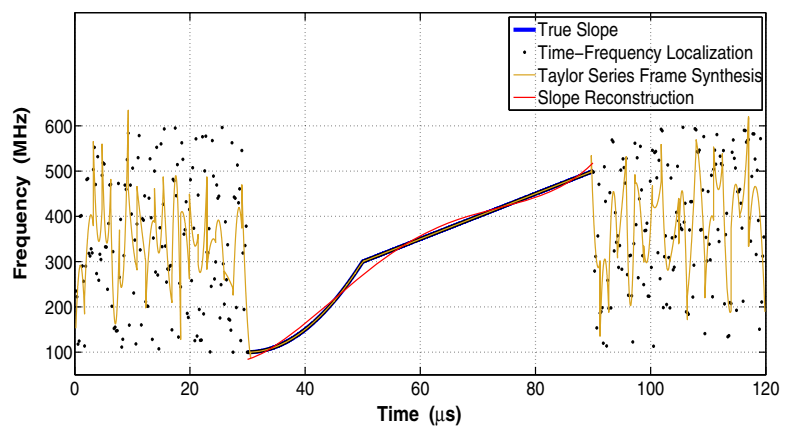

(h) 
4Figure 7. LPI radar scenario simulation and analysis using the proposed method. Analysis of AWGN, Unmodulated, LFM, NLFM, FMCW and ArFM signals at $-8 \mathrm{~dB}$ SNR in a digital receiver with $500 \mathrm{MHz}$ bandwidth, with noise level at $-75 \mathrm{dBm}$, using MFB, $\mathbf{A}_{2}^{\left(512 \times N_{2}\right)}$. (a) Analysis under no-signal condition with only real AWGN at $-75 \mathrm{dBm}$ in a $500 \mathrm{MHz}$ bandwidth digital receiver. (b) Unmodulated signal analysis. $F=500 \mathrm{MHz}$, $\hat{F}=499.97 \mathrm{MHz}, \quad T=10 \mu \mathrm{s}, \hat{T}=9.82 \mu \mathrm{s}$. (c) LFM analysis. $F=350 \mathrm{MHz}, \quad \hat{F}=349.90 \mathrm{MHz}, \quad B=500 \mathrm{MHz}, \quad \hat{B}=$ $499.57 \mathrm{MHz}, T=25 \mu \mathrm{s}, \hat{T}=24.98 \mu \mathrm{s}$. (d) Analysis of quadratic FM with concave, negative slope. $F=225 \mathrm{MHz}, \hat{F}=$ $224.99 \mathrm{MHz}, \quad B=3 \mathrm{MHz}, \quad \hat{B}=3.01 \mathrm{MHz}, \quad T=100 \mu \mathrm{s}, \quad \hat{T}=$ $99.87 \mu \mathrm{s}$. (e) Analysis of quadratic FM with convex, positive slope. $F=300 \mathrm{MHz}, \quad \hat{F}=300.44 \mathrm{MHz}, \quad B=200 \mathrm{MHz}, \quad \hat{B}=$ $199.14 \mathrm{MHz}, T=30 \mu \mathrm{s}, \hat{T}=29.74 \mu \mathrm{s}$. (f) LFMCW analysis. $F=350 \mathrm{MHz}, \quad \hat{F}=350.27 \mathrm{MHz}, \quad B=500 \mathrm{MHz}, \quad \hat{B}=$ 499.41 MHz, $T=100 \mu \mathrm{s}, \hat{T}=99.88 \mu \mathrm{s}, \quad P W=400 \mu \mathrm{s}, \quad P \hat{W}=$ $399.54 \mu \mathrm{s}$. (g) Analysis of FMCW with linear, positive slope \& non-linear, negative slope. $F=400 \mathrm{MHz}, \hat{F}=400.11 \mathrm{MHz}, B=$ $100 \mathrm{MHz}, \hat{B}=99.94 \mathrm{MHz}, T_{u p}=50 \mu \mathrm{s}, \hat{T}_{u p}=49.96 \mu \mathrm{s}, T_{d n}=$ $25 \mu \mathrm{s}, \hat{T}_{d n}=24.93 \mu \mathrm{s}, P W=150 \mu \mathrm{s}, P W=149.80 \mu \mathrm{s}$. (h) Arbitrary FM analysis. $F=300 \mathrm{MHz}, \quad \hat{F}=301.04 \mathrm{MHz}, \quad B=$ $400 \mathrm{MHz}, \hat{B}=433.96 \mathrm{MHz}, T=60 \mu \mathrm{s}, \hat{T}=59.97 \mu \mathrm{s}$.

and arbitrary FM SOIs existing simultaneously over a large dynamic range.

\section{Conclusion}

The present work addresses a prominent problem in the domain of EW signal processing and system design. Suitability of linearity and continuity assumptions at subframe processing stage and continuity assumption at frame processing stage has been analytically explored and demonstrated. It is worthwhile to recognize the applicability and validity of these assumptions with respect to present day EW systems and real-life threat radar scenarios. Consistent detection, estimation and classification performances and SOI reconstruction capability are shown for candidate signal set in the likely LPI SNR regime, under stringent conditions. Performance under null hypothesis has been studied extensively. Capability to synthetically reconstruct IF of SOI and its usefulness are demonstrated. The proposed method facilitates meeting specific operational objectives by corresponding design of MFBs. New signal types can be included in classification hierarchy with corresponding model definition and cost function formulation. Scope for improvement exists in all stages of analysis. MFB design, weighted projection schemes, signal detection algorithms, classificiation methods, etc. are all open opportunities. The proposed architecture focuses on core signal processing methodology for analysis of SOIs with continuous-time IF structure to establish the stated concepts.

\section{Acknowledgements}

Authors are thankful to The Director, Indian Institute of Science, Bangalore, The Director, Naval Physical and Oceanographic Laboratory, Kochi, and The Director, Defence Avionics Research Establishment, Bangalore, for the academic and organizational support. The present work is based on the academic project undertaken by A R Sachin as a part of his Master of Engineering (Telecommunications) Degree program, under the guidance of Prof K V S Hari, in association with Dr Sooraj K Ambat, during 2011-2013, in Statistical Signal Processing Lab, Department of Electrical Communication Engineering, Indian Institute of Science, Bangalore.

\section{References}

[1] Tsui J B 2004 Digital techniques for wideband receivers, 2nd edn. Scitech, North Carolina, USA

[2] Cohen L 1995 Time-frequency analysis. Prentice Hall, New Jersey, USA

[3] M/s Thales Group 2012 Product Datasheet VIGILE advanced naval R-ESM/ELINT systems. www.thalesgroup.com

[4] M/s Selex ES 2014 Product Datasheet SAGE advanced digital ESM.www.selex-es.com

[5] M/s Saabgroup 2015 Product Datasheet SIRIUS airborne SigInt system. www.saabgroup.com

[6] M/s Rockwell Collins 2009 Product Datasheet CS3045 airborne ELINT/ESM subsystem. www.rockwellcollins.com/ ewsigint

[7] Pace P E 2004 Detecting and classifying low probability of intercept radar. Artech House Inc, Massachusetts, USA

[8] Taboada F L 2002 Detection and classification of LPI radar signals using parallel filter arrays and higher order statistics. MS Project Thesis. USA: Naval Post Graduate School, US Navy

[9] Raju V V S R N, Pavan Kumar R, Singh A K and Subba Rao K 2012 LPI radar signal detection, identification and classification using digital receiver, Second international Conference on Electronic Warfare, EWCI-2012, Bangalore, India, pp. 522-528

[10] Lunden J and Koivunen V 2007 Automatic radar waveform recognition. IEEE J. Sel. Top. Signal Process. 1(1): 124-136

[11] Lunden J, Terho L and Koivunen V 2005 Classifying pulse compression radar waveforms using time frequency distributions. In: Proceedings of the Conference on Info Sciences and Systems, The Johns Hopkins University, March 2005

[12] Cornu C, Ioana C, and Quinquis A 2004 Characterization of LPI waveforms using polynomial phase signal modelling. In: proceeding of the International Conference on Radar Systems, Toulouse, France. [Online]. Available: https://hal. archives-ouvertes.fr/hal-00518788

[13] Geroleo F G and Brandt-Pearce M 2012 Detection and estimation of LFMCW radar signals. IEEE Trans. Aerosp. Electron. Syst. 48(1): 405-418

[14] Stoica P and Moses R 2005 Spectral analysis of signals. Prentice-Hall, New Jersey, USA 
[15] Stoica P and Selen Y 2004 Model-order selection. IEEE Signal Process. Mag. 1053-5888/04:36-47

[16] Hamschin B, Clancy J, Grabbe M, Fortier M and Novak J 2014 In: Proceedings of the Passive detection, characterization, and localization of multiple LFMCW LPI signals. IEEE Radar Conference, pp. 537-543

[17] Oh D and Lee J H 2015 Low-complexity range-azimuth FMCW radar sensor using joint angle and delay estimation without SVD and EVD. IEEE Sens. J. 5(9): 4799-4811

[18] Zhou Y, Wang X, Tian Y and Zhou D 2015 A novel timefrequency atomic dictionary for radar intra-pulse modulation signal sparse representation. In: Proceedings of IEEE Microwave Conference (APMC), Asia Pacific, vol. 1, pp. 1-3

[19] Guner K K, Erkmen B, Gulum T O, Yasin Erdogan A, Yildirim T and Ata L D 2016 Implementation aspects of Wigner-Hough Transform based detectors for LFMCW signals. In: Proceeding of the $39^{\text {th }}$ IEEE International Conference on Telecommunications and Signal Processing, pp. 441-444

[20] Rossouw van der Merwe J, du Plessis W P, Maasdorp F D V and Cilliers J E 2016 Introduction to low probability of recognition to radar system classification. In: Proceedings of the IEEE Radar Conference, pp. 1-5
[21] Neves S R, de Oliveira A, Serra R, Segadilha L E, Monteiro F and Lopez J M 2016 Using wavelet packets to analyze FM LPI radar signals. In: Proceeding of the IEEE Sensor Array and Multichannel Signal Processing Workshop (SAM), pp. 1-4

[22] Lopez-Risueno G and Grajal J 2006 Multiple signal detection and estimation using atomic decomposition and EM. IEEE Trans. Aerosp. Electron. Syst. 42(1): 84-102

[23] Lopez-Risueno G, Grajal J, Omar A, Ojeda Y, Sanz-Osorio A and Moreno J A 2003 Two digital receivers based on timefrequency analysis for signal interception. In: Proceedings of the conference IEEE Radar

[24] Lopez-Risueno G, Grajal J and Ojeda Y 2003 Atomic decomposition based radar complex signal interception. IEEE Proc. Radar Sonar Navig. 150(4): 323-331

[25] Skolnik M L 2001 Introduction to radar systems, 3rd edn. Tata Mcgraw-Hill, Delhi, India

[26] Sayed A H 2008 Adaptive filters. Wiley-IEEE Press, New Jersey, USA

[27] Edwin K P Chong and Stanislaw H Zk 2001 An introduction to optimization, 2nd edn. Wiley, New York, USA 